\title{
Atenção Básica em Saúde: comparação entre PSF e UBS por estrato de exclusão social no município de São Paulo
}

\author{
Primary Health Care: a comparison of PSF \\ and UBS units per stratum of socially excluded \\ users in the city of São Paulo
}

Paulo Eduardo Elias 1,2

Clara Whitaker Ferreira 1

Maria Cecília Góis Alves 3

Amélia Cohn 1,2

Vanessa Kishima 4

Álvaro Escrivão Junior 1,4

Adriana Gomes 1,5

Aylene Bousquat 1,5

${ }^{1}$ Centro de Estudos de Cultura Contemporânea. Rua Airosa Galvão 64,

Água Branca, 05002-070 São Paulo SP.

cedec@cedec.org.br

2 Departamento de

Medicina Preventiva,

FMUSP.

3 Instituto de Saúde, Secretaria de Estado da Saúde de São Paulo.

4 Fundação Getúlio

Vargas de São Paulo.

5 Programa de Mestrado

em Saúde Coletiva,

Universidade Católica

de Santos.
Abstract Within the baseline studies of the PROESF, this article aims to compare the assistance modalities PSF and the traditional UBS per stratum of socially excluded users in São Paulo. The study is considering the opinions of users, health professionals and managers. The tool used for this comparison was the Primary Care Assessment Tool (PCAT), which contemplates eight dimensions of primary care. The questionnaire was answered by several professionals of the 384 primary care units. The best evaluated PSF and UBS units (Basic Care Index-BCI) were selected from each of the three groups and socially excluded stratums. An opinion survey was carried out in each one of these units. The study resulted in scores of perception of users, managers and health professionals in each modality, focusing the eight dimensions of primary care. With exception to one stratum, the opinions of managers and health professionals were found very similar with regard to all studied dimensions and, compared with the opinion of users, they were always better, especially as refers to orientation towards the family and the community. The comparison of PSF and UBS with regard to the different socially excluded stratums shows no differences between the opinions of managers and professionals. The BCI found based on the opinion of users however was higher for the PSF than for the traditional UBS units.

Key words Primary health care, PSF, Assessment of primary care
Resumo Dentro dos Estudos de Linha de Base do Proesf, este artigo compara as modalidades assistenciais Programa de Saúde da Família (PSF) e Unidade Básica de Saúde (UBS) tradicional por estrato de exclusão social no Município de São Paulo, considerando as opiniões de usuários, profissionais de saúde e gestores. O instrumento utilizado, Primary Care Assessment Tool (PCAT), contempla oito dimensões da atenção básica. O questionário foi respondido por diversos profissionais de 384 unidades de saúde. Foram escolhidas as unidades PSF e UBS mais bem avaliadas (Indice de Atenção Básica - IAB) de cada um dos três agrupamentos de estratos de exclusão social. Em cada unidade foi realizado inquérito de opinião com usuários, num total de 1.117 questionários. O estudo resultou na elaboração de escores de percepção de usuários, gestores e profissionais para cada modalidade, abrangendo as oito dimensões da atenção básica. À exceção de um estrato, as percepções dos gestores e profissionais são muito semelhantes para todas as dimensões estudadas e, em comparação às dos usuários, são sempre melhores, especialmente no enfoque familiar e orientação comunitária. A comparação entre PSF e UBS nos diferentes estratos de exclusão social não apresenta diferenças para profissionais e gestores. Contudo, o $I A B$ aferido por meio dos usuários é mais elevado nas Unidades PSF do que nas UBS tradicionais. Palavras-chave Atenção básica, PSF, Avaliação da atenção básica 


\section{Introdução}

O Programa de Saúde da Família (PSF) emerge em 1994 e desde o final da década de 1990 vem sendo assumido pelo Ministério da Saúde como a principal estratégia de organização da atenção básica à saúde no país. Em 20041, o PSF abrangia 80,2\% (4.492) dos municípios brasileiros, cobrindo apenas 36,4\% (63.655.488) da população.

A desproporção entre o percentual da população cadastrada e o de municípios abrangidos denota a disseminação do Programa nos municípios de pequeno e médio porte e também espelha o maior constrangimento para a consolidação do PSF como modalidade organizativa da atenção básica no País: atingir coberturas universais das populações residentes nos municípios de grande porte, notadamente naqueles com mais de 500.000 habitantes ${ }^{2}$.

$\mathrm{Na}$ sua maioria, estes municípios possuem um histórico de oferta de serviços de saúde que se traduz na existência de redes com experiências acumuladas em outras modalidades da atenção básica. É exatamente nestes municípios, nos quais o PSF não assume de pronto sua feição de ampliação de acesso, que esta modalidade vem tendo maior dificuldade em se apresentar como estruturante da atenção básica. Desta forma, torna-se importante indagar em que aspectos a implantação do PSF nas metrópoles pode contribuir para o acesso à atenção básica à saúde universal e igualitária e também quais são as vantagens comparativas desta nova e das demais modalidades já incorporadas na rede de serviços.

Para contribuir neste debate, o estudo das modalidades de atenção básica vigentes na metrópole paulista certamente adquire relevância nacional. Apesar de o quadro paulistano diferir do encontrado na grande maioria das outras grandes cidades, uma vez que o PSF foi concebido como estratégia de reorganização da atenção à saúde, o porte e a tradição de sua rede possibilita a coexistência de diversas lógicas assistenciais, propiciando o estudo dos constrangimentos e das potencialidades destas modalidades.

Ademais, São Paulo aglutina as características mais marcantes das metrópoles dos países capitalistas periféricos, dentre as quais se destacam a economia dinâmica e inserida no circuito internacional, a especialização financeira, o papel determinante das telecomunicações para centralização das funções de comando e a presença de um forte padrão de exclusão social que adquire uma feição espacial, caracterizando um espaço urbano marcadamente heterogêneo ${ }^{3,4}$.

No caso da saúde, essas heterogeneidades se traduzem no seu dinamismo, evidenciado na alta concentração tecnológica, na cobertura de $47,2 \%$ da população por Planos de Saúde 5 , na grande rede de serviços públicos e privados, somando mais de 5.000 estabelecimentos de saúde, dos quais 183 hospitais 6 , e também pelas barreiras impostas a parcelas importantes de seus habitantes no acesso à assistência à saúde, principalmente na atenção básica.

Desde 2001, toda a rede de atenção básica encontra-se sob o comando municipal e atualmente a Secretaria Municipal de Saúde (SMS) se estrutura administrativamente em cinco Coordenadorias Regionais de Saúde (Leste, Sudeste, Centro-Oeste, Sul e Norte), às quais estão vinculadas 384 unidades de saúde, sendo 173 (45\%) com PSF. Vale sublinhar que as diretrizes gerais para o funcionamento dos serviços de atenção básica são estabelecidas no nível central da SMS, pela Coordenadoria Geral de Atenção Básica. A implantação do PSF priorizou as regiões nas quais a renda familiar era de até 5 salários mínimos. A forma operacional utilizada baseava-se na parceria da SMS com instituições privadas não lucrativas, com tradição de atuação na área de saúde no município de São Paulo ${ }^{7}$.

As unidades PSF contam com Equipes de Saúde da Família (ESF) em conformidade com as normas do MS e também obedecem ao preceito da delimitação de área de abrangência com adstrição de clientela. Já as UBS contam em suas equipes com médicos (clínicos, pediatras e ginecologista-obstetras), enfermeiros, dentistas, auxiliares de enfermagem e pessoal de apoio técnico. Há também a presença de médicos de diversas especialidades (dentre os quais oftalmologistas, dermatologistas, cardiologistas, pneumologistas), distribuídos irregularmente pelas unidades. A demanda atendida se apresenta como espontânea e/ou encaminhada por outros serviços. Neste caso não há adstrição de clientela, e a delimitação da área de abrangência se refere exclusivamente às ações de vigilância à saúde.

Para abordar as questões assinaladas, há necessidade de se estabelecer um marco referencial de análise que resulte em um instrumento suficientemente abrangente e ágil para o estudo da atenção básica. Desta forma, optou-se por utilizar o referencial proposto por Starfield8, 
que se desdobra em dimensões de avaliação da atenção básica à saúde, denominadas de: acessibilidade, porta de entrada, elenco de serviços, vínculo, coordenação, orientação familiar, orientação comunitária e formação profissional, com as correspondentes definições 9 :

Acessibilidade - presença ou ausência de barreiras financeiras, organizacionais, e/ou estruturais para se conseguir atenção básica à saúde;

Porta de entrada - o grau dos serviços em se constituírem como porta de entrada para os outros níveis de atenção, exceto em emergências;

Vínculo ou longitudinalidade - diz respeito à utilização regular do estabelecimento pela população e o foco da equipe na população adstrita;

Elenco de serviços - contempla o adequado fornecimento de um rol mínimo de serviços adequados às necessidades da população adstrita;

Coordenação ou integração de serviços contempla a facilidade em acessar os demais níveis de atenção e a integração com os serviços de outros setores sociais;

Centralidade na família - na assistência, contemplar o contexto e a dinâmica familiar;

Orientação para a comunidade - a capacidade da atenção primária em reconhecer e responder às necessidades da comunidade e promover ações intersetoriais nas ações comunitárias;

Formação profissional - envolve o conteúdo e a extensão da capacitação da equipe de atenção básica à saúde.

Segundo diversos autores, os sistemas de saúde que cumprem os preceitos contidos nestas dimensões apresentam melhor desempenho10, 11, 12. Segundo Macinko13, a efetivação dos postulados destas dimensões é associada a melhores indicadores de saúde, mesmo após se realizar o controle de variáveis como renda per capita, uso de tabaco e álcool e número de médicos.

Dessa forma, as dimensões da atenção básica propostas por Starfield se revestem de especial importância para a análise das potencialidades das duas principais modalidades de atenção básica vigentes na metrópole paulista.

\section{Objetivos}

Comparar as modalidades PSF e UBS tradicional por estrato de exclusão social, considerando as dimensões da atenção básica propostas por Starfield e identificar as convergências e divergências de opinião de usuários, profissionais de saúde e gestores por unidade de saúde.

\section{Metodologia}

Para a comparação das duas modalidades de assistência prevalecentes na rede de atenção básica no município de São Paulo, optou-se pela utilização do instrumento Primary Care Assessment Tool (PCAT)R, elaborado por Bárbara Starfield e James Macinko. Este instrumento foi adaptado e validado para o Brasil por Almeida e Macinko por meio de sua aplicação em município de médio porte no Estado do Rio de Janeiro14, 15. O questionário, abrangendo os mesmos tópicos, apresenta versões dirigidas a diferentes informantes nos serviços de saúde (profissionais e usuários), de modo a propiciar o diálogo entre as opiniões dos dois segmentos.

O PCAT se estrutura em oito blocos, cada um abrangendo as dimensões propostas por Starfield para a análise de modalidade de assistência na atenção básica. $\mathrm{O}$ instrumento é composto por cerca de 100 perguntas, distribuídas pelos blocos correspondentes às dimensões da atenção básica. Cada pergunta contém sete possibilidades de resposta (nunca, quase nunca, algumas vezes, muitas vezes, quase sempre, sempre, não sabe), e a sua aferição se dá por meio de escala, na qual 0 (zero) corresponde ao pior desempenho e 5 ao melhor. A média aritmética simples das questões de cada bloco apura o índice daquela dimensão, e por sua vez, a média aritmética simples destes leva ao Índice de Atenção Básica (IAB) da unidade de saúde.

O campo da pesquisa se desenvolveu em duas fases: na primeira, foram apuradas as opiniões de gerentes, médicos e enfermeiros das 384 unidades da rede municipal de atenção básica; na segunda foram realizados inquéritos com usuários de unidades selecionadas.

$\mathrm{Na}$ primeira fase, a partir do cadastro de pessoal fornecido pela Secretaria Municipal de Saúde (SMS), foram enviados três questionários para cada unidade de saúde, a serem respondidos pelo gerente, por médico e enfermeiro, estes últimos escolhidos por sorteio dentre os profissionais lotados na unidade. Havia também a possibilidade de resposta ao questionário via versão eletrônica, disponibilizada por Internet no portal RAPS (Rede de Análise de Políticas de Saúde). Para poder dar esta forma de resposta, cada entrevistado recebia junto com o questionário uma identificação e senha para acesso a este portal. Após o preenchimento do questionário na versão impressa, os participantes o enviavam em envelope lacrado para a Coordenação Regional de Saúde, de onde 
eram recolhidos pelos pesquisadores. Todos aqueles que aderiram à pesquisa receberam $\mathrm{e}$ assinaram Termo de Consentimento Informado.

A todo entrevistado era solicitado que se auto-avaliasse quanto à confiabilidade de suas respostas. Foram excluídos da análise quatro questionários nos quais os entrevistados referiram baixa confiabilidade. Foram excluídas da análise 41 UBS nas quais os questionários não foram respondidos pelo gerente e/ou por nenhum dos dois profissionais da equipe de saúde. Nas 343 unidades incluídas foram calculados os indicadores por dimensão e o IAB para cada questionário, resultando em três escores de profissionais. A média aritmética dos escores do médico e do enfermeiro constituiu o indicador de avaliação do profissional técnico da unidade. O IAB de cada unidade foi calculado procedendo-se à média aritmética dos indicadores de avaliação do gerente e dos profissionais. Obteve-se, assim, a distribuição dos IABs das unidades de saúde.

Para a seleção das unidades de saúde dos modelos de assistência a serem comparados (PSF e UBS tradicional) foram considerados três agrupamentos de estratos de exclusão social, conforme estudo de Bousquat 16 , que identificou um padrão de implantação do PSF nitidamente distinto por referência aos estratos de exclusão social no Município de São Paulo. Estes estratos foram definidos a partir do Índice de Exclusão/Inclusão Social (IEX) dos Distritos Administrativos (DAs) referidos ao ranking do Mapa da Exclusão Social do Município ${ }^{17}$. Este índice é um indicador composto construído a partir de 47 variáveis agregadas em quatro grandes áreas: autonomia, qualidade de vida, desenvolvimento humano e eqüidade. A cada um desses indicadores foram atribuídas notas decimais negativas e positivas, variando de -1 a 1 , sendo o zero definido como o padrão básico de inclusão social. Os distritos foram classificados nesses intervalos em função da distância positiva ou negativa do padrão. A faixa de variação (-1 a 1) foi dividida, levando à identificação de 8 estratos de exclusão/inclusão social no município.

As 343 Unidades de Saúde foram redistribuídas segundo estes agrupamentos (1 e 2; 3 e $4 ; 5$ a 8 ) e, para cada estrato resultante, foram selecionadas duas unidades de saúde: a com melhor escore dentre as do PSF e a com melhor escore dentre as UBS tradicionais, resultando na escolha de 6 unidades de saúde. Dado o escopo do estudo, as unidades de saúde nas quais coexistiam as modalidades PSF e tradicional (unidades mistas) foram excluídas do rol das unidades a serem selecionadas.

Na segunda fase, em cada uma das seis unidades escolhidas, foi sorteada uma amostra dos usuários que passaram por consulta médica nos 10 dias úteis de duração da pesquisa. Foi planejada a realização de 196 entrevistas por unidade e, prevendo-se uma taxa de não resposta de $20 \%$, calculou-se o sorteio de 245 usuários. O sorteio foi realizado a partir da lista de pacientes agendados e vagas previstas para consultas sem agendamento. $\mathrm{O}$ tamanho da amostra foi calculado por meio da expressão algébrica (Kish18):

$\mathrm{n}=\frac{s_{y}^{2}}{\operatorname{var}(\bar{y})}$,

onde $s_{y}{ }^{2}$ é a variância por elemento de " $y$ ", resultado da avaliação, estimado em 1,00, com base em dados do estudo de Macinko14; $\operatorname{var}(\bar{y})=$ $(d / t)^{2}$ é a variância a ser tolerada para a média de " $y$ ", sendo $d$ o erro de amostragem, fixado em 0,14 , e $t$ o valor da curva normal correspondente ao nível de confiança de 95\% $(t=1,96)$.

As entrevistas com os usuários das unidades de saúde foram realizadas simultaneamente nas unidades por entrevistadores treinados. O PCAT foi disponibilizado em meio eletrônico no equipamento palm-top, com sistema de checagem automática de preenchimento para controlar erros de digitação.

Foram entrevistados 1.117 usuários nas seis unidades de saúde selecionadas. Do total sorteado, foram excluídos os usuários de primeira consulta na unidade. O percentual de não resposta foi de $14,7 \%$ da amostra.

Para a análise dos dados foi utilizado o Programa SPSS, tendo sido realizados testes $t$ para comparação de médias e testes de qui-quadrado para estudo de associação com dados categóricos, nível de significância de 5\%.

\section{Resultados e discussão}

Uma breve comparação entre as unidades de saúde de cada estrato em relação às características dos usuários sorteados indica a presença de diferenças estatisticamente significantes entre as médias das idades nos estratos $1 / 2$ e $5 / 8$, sendo que os usuários das unidades PSF apresentam idade média mais alta. Em relação à distribuição por sexo, há diferença entre as uni- 
dades no estrato 3/4. A maioria dos entrevistados da UBS é do sexo masculino, conforme se observa na tabela 1 .

A tabela 2 mostra os escores de percepção dos usuários obtidos para cada uma das dimensões e o Índice de Atenção Básica, acompanhado do correspondente intervalo de confiança, em cada unidade. Estão indicados em negrito os escores que se mostraram maiores (diferenças estatisticamente significantes) na comparação entre os dois tipos de unidades, dentro de cada estrato.

Chama a atenção, de início, que em todos os estratos as estimativas dos Índices de Atenção Básica das Unidades PSF são mais altos do que os das UBS tradicionais, sempre com diferenças estatisticamente significantes. Ao considerarmos somente as Unidades PSF, observamos diferenças entre os índices, o maior deles correspondendo ao estrato de menor exclusão. Nas unidades tradicionais, a avaliação é uniforme. Observamos ainda que os valores se situam todos entre 3 e 4 , o que significa que as situa- ções descritas ocorrem “muitas vezes”. No entanto, nas Unidades PSF os escores estão mais próximos de 4, ou seja, as situações descritas ocorrem "quase sempre"; e nas Unidades tradicionais, mais próximos de 3 , o que corresponde a "algumas vezes".

Na dimensão "Acessibilidade”, a unidade PSF do estrato $1 / 2$ obteve um escore mais alto, ao contrário do estrato $3 / 4$, em que a UBS tradicional obteve o valor mais alto. Cabe ressaltar que a avaliação da acessibilidade foi regular: os índices são próximos de 2,5 em todas as unidades, o que significa que as situações que definem uma boa acessibilidade ocorrem "algumas vezes", ou no máximo "muitas vezes".

Já no que diz respeito à avaliação do "Vínculo", verifica-se uma diferença significativa entre a unidade PSF e a UBS somente no estrato de maior exclusão, quando na realidade o esperado seria haver diferenças em todos os estratos, uma vez que o PSF tem no seu ideário o estabelecimento de vínculo entre a família e os

\section{Tabela 1}

Número de entrevistas, idade média e proporção de usuários do sexo masculino e respectivos intervalos de confiança (IC), por unidade.

\begin{tabular}{|c|c|c|c|c|c|c|}
\hline & \multicolumn{2}{|c|}{ Estrato $1 / 2$} & \multicolumn{2}{|c|}{ Estrato 3 / 4} & \multicolumn{2}{|c|}{ Estrato 5 / 8} \\
\hline & PSF & UBS & PSF & UBS & PSF & UBS \\
\hline No. entrevistas & 184 & 201 & 165 & 211 & 159 & 197 \\
\hline Idade - Média & $32,9^{*}$ & $25,6^{*}$ & 33,9 & 36,8 & $39,6^{\star}$ & $32,8^{*}$ \\
\hline IC & {$[29,5-36,3]$} & {$[22,9-28,4]$} & {$[30,0-37,8]$} & {$[32,9-38,3]$} & {$[36,4-43,1]$} & {$[29,5-36,1]$} \\
\hline Sexo masc. - Proporção & 29,9 & 28,9 & $33,3^{\star}$ & $55,0^{\star}$ & 28,3 & 19,8 \\
\hline IC & {$[23,2-36,6]$} & {$[22,5-35,2]$} & {$[26,1-40,6]$} & {$[48,2-61,7]$} & {$[21,2-28,0]$} & {$[14,2-25,4]$} \\
\hline
\end{tabular}

${ }^{*} \mathrm{p}<0,05$

\section{Tabela 2}

Escores atribuídos pelos usuários segundo dimensão da atenção básica, por estrato e tipo de unidade.

\begin{tabular}{llllllc}
\hline Dimensão & \multicolumn{2}{c}{ Estrato 1 / } & \multicolumn{2}{c}{ Estrato 3 / } & \multicolumn{2}{c}{ Estrato 5 / 8 } \\
& PSF & UBS & PSF & UBS & PSF & UBS \\
\hline Acessibilidade & $\mathbf{2 , 6 6}$ & 2,5 & 2,44 & $\mathbf{2 , 8 9}$ & 2,6 & 2,53 \\
Porta de entrada & 4,13 & $\mathbf{4 , 4 7}$ & $\mathbf{4 , 4 8}$ & 3,63 & $\mathbf{4 , 3 9}$ & 3,34 \\
Vínculo & 4 & $\mathbf{4 , 1 4}$ & 4,01 & 3,99 & 4,26 & 4,29 \\
Serviços & 4,21 & 4,23 & $\mathbf{4 , 3 3}$ & 4,15 & 4,31 & 4,26 \\
Coordenação & 4,44 & 4,38 & $\mathbf{4 , 1 2}$ & 3,9 & $\mathbf{4 , 3 7}$ & 3,58 \\
Enfoque familiar & $\mathbf{2 , 5 2}$ & 1,4 & $\mathbf{2 , 2 9}$ & 1,69 & $\mathbf{3 , 1 6}$ & 1,69 \\
Orientação comunitária & $\mathbf{2 , 5 9}$ & 1,7 & $\mathbf{2 , 9 9}$ & 1,63 & $\mathbf{3 , 3 3}$ & 1,44 \\
Profissionais de saúde & $\mathbf{4 , 0 9}$ & 3,48 & 4,22 & $\mathbf{4 , 3 9}$ & $\mathbf{4 , 7}$ & 4,35 \\
Índice Atenção Básica & $\mathbf{3 , 5 8}$ & 3,29 & $\mathbf{3 , 6 1}$ & 3,28 & $\mathbf{3 , 8 9}$ & 3,19 \\
Intervalo de confiança & {$[3,51-3,65]$} & {$[3,22-3,35]$} & {$[3,55-3,67]$} & {$[3,20-3,36]$} & {$[3,80-3,97]$} & {$[3,11-3,27]$} \\
\hline
\end{tabular}

Negrito: $\mathrm{p}<0,05$. 
profissionais da unidade PSF. De qualquer forma, a dimensão do vínculo é bem percebida de forma geral, com escores superiores a $4 \mathrm{em}$ todas as unidades de todos os estratos, significando que o estabelecimento do vínculo ocorre "quase sempre".

Comportamentos semelhantes apresentam as dimensões "Enfoque familiar" e "Orientação comunitária”, com escores mais altos nas unidades PSF, com diferenças significativas em todos os estratos. Embora seja um resultado esperado, pois as duas dimensões são um componente essencial do PSF, chama a atenção o fato de os valores atribuídos a elas serem baixos: nas unidades PSF, situam-se próximos de 2,5 nos estratos $1 / 2$ e $3 / 4$, e de 3 no estrato 5/8. Já nas UBS, são as dimensões que obtiveram os escores mais baixos: abaixo de 2 em todas as unidades, significando que, na avaliação dos usuários, o enfoque familiar e a orientação comunitária ocorrem "quase nunca" nessas unidades.

Quanto à avaliação da dimensão "Serviços oferecidos", houve diferença entre as unidades somente no estrato $3 / 4$, em que a unidade PSF obteve um escore mais elevado. Neste caso, os escores foram também todos superiores a 4 .

Já no que diz respeito à dimensão "Coordenação dos cuidados”, obtiveram escores mais altos as unidades PSF dos estratos $3 / 4$ e 5/8, enquanto na dimensão "Profissionais de saúde", verificam-se diferenças estatisticamente significantes entre as unidades de saúde em todos os estratos: nos estratos $1 / 2$ e 5/8 a favor do PSF; no estrato $3 / 4$ a favor da UBS.

Até o momento, foi focada somente a opinião dos usuários. Interessante, no entanto, é comparar esta percepção com a dos gestores e dos profissionais de saúde, em que pese a natureza de as informações serem distintas dados os procedimentos metodológicos adotados. A sistematização desses dados encontra-se na tabela 3. Ambos mostram, para cada dimensão, o escore médio da avaliação dos usuários, o escore do gestor da unidade e o escore médio dos dois profissionais entrevistados.

De imediato, chama a atenção que a opinião dos gestores e dos profissionais é muito semelhante, para todas as dimensões, à exceção do estrato $5 / 8$, no qual os gestores atribuíram escores mais elevados que os profissionais de saúde. Note-se, ainda, que as percepções dos gestores e dos profissionais são sempre melhores que as dos usuários, com apenas duas exceções: a dimensão "Acessibilidade" no estrato $3 / 4$ e a dimensão "Profissionais de saúde" na UBS do estrato $1 / 2$. Não obstante, é a dimensão "Acessibilidade" a que recebe a pior opinião dos gestores e profissionais de saúde, com isso aproximando-se da percepção dos usuários.

Finalmente, chama a atenção os altos escores atribuídos pelos gestores e profissionais às dimensões "Enfoque familiar" e "Orientação comunitária" nas Unidades PSF, embora sejam as dimensões que obtiveram os índices menores entre os usuários. Trata-se de um resultado esperado se levarmos em conta que são duas dimensões bastante enfatizadas no Programa de Saúde da Família e que dificilmente seriam malavaliadas pelos próprios gestores e profissionais encarregados de implantá-las nos serviços.

\section{Considerações finais}

Segundo o estudo, a população que freqüenta as unidades de saúde é relativamente jovem, ambos os sexos em idade produtiva e as mulheres em idade fértil, sugerindo especificidades na oferta e organização da assistência à saúde, bem como em relação aos juízos de valor que expressam.

Ao se cotejarem os resultados obtidos temse que considerar o desenho do estudo que, ao buscar avaliar as potencialidades dos dois modelos de atenção básica, selecionou, para cada um deles, as unidades com melhor desempenho na opinião dos profissionais e gestores, nos diferentes estratos de exclusão. Neste sentido, o resultado geral bastante satisfatório alcançado pelas seis unidades estudadas reitera as potencialidades para a estruturação de uma rede de atenção primária voltada às expectativas de usuários e profissionais de saúde, fato de não menor importância se considerada a situação bastante diversa da média das unidades de atenção básica no município, que muito têm a caminhar na direção das estudadas e o enfoque desfavorável que os meios de comunicação reservam ao Sistema Único de Saúde (SUS).

Entretanto, a opinião dos usuários é comparativamente mais favorável ao PSF em todos os estratos de exclusão, sendo os índices alcançados inversamente proporcionais aos das situações de exclusão, isto é, quanto maior a exclusão, menor o índice de aprovação ao PSF. Este achado da pesquisa contraria afirmações de documentos oficiais e de administradores sobre a pretensa aceitação uniforme do PSF pela população e especialmente pela população situada nos estratos de baixa renda. 
Tabela 3

Distribuição dos escores atribuídos pelos usuários, gestores e profissionais, segundo dimensão da atenção básica e unidade.

\begin{tabular}{|c|c|c|c|c|c|c|c|c|c|}
\hline \multirow[t]{2}{*}{ Entrevistado } & \multicolumn{9}{|c|}{ Dimensão } \\
\hline & $\begin{array}{l}\text { Acessibi- } \\
\text { lidade }\end{array}$ & $\begin{array}{l}\text { Porta de } \\
\text { entrada }\end{array}$ & Vínculo & Serviços & $\begin{array}{l}\text { Coorde- } \\
\text { nação }\end{array}$ & $\begin{array}{l}\text { Enfoque } \\
\text { Familiar }\end{array}$ & $\begin{array}{l}\text { Orientação } \\
\text { comunitária }\end{array}$ & $\begin{array}{l}\text { Profis- } \\
\text { sionais } \\
\text { de saúde }\end{array}$ & $\begin{array}{l}\text { Índice } \\
\text { Atenção } \\
\text { Básica }\end{array}$ \\
\hline \multicolumn{10}{|l|}{ Estrato $1 / 2$} \\
\hline \multicolumn{10}{|l|}{ Unidade 106 PSF } \\
\hline Usuários & 2,66 & 4,13 & 4,00 & 4,21 & 4,44 & 2,52 & 2,59 & 4,09 & 3,58 \\
\hline Gestor & 3,44 & 5,00 & 4,71 & 4,75 & 4,74 & 5,00 & 4,57 & 4,80 & 4,62 \\
\hline Profissionais & 3,33 & 5,00 & 4,86 & 4,94 & 4,65 & 5,00 & 4,50 & 4,30 & 4,57 \\
\hline \multicolumn{10}{|c|}{ Unidade 325 UBS } \\
\hline Usuários & 2,50 & 4,47 & 4,14 & 4,23 & 4,38 & 1,40 & 1,70 & 3,48 & 3,29 \\
\hline Gestor & 3,22 & 5,00 & 3,14 & 4,38 & 4,53 & 3,50 & 4,57 & 2,60 & 3,86 \\
\hline Profissionais & 2,94 & 5,00 & 3,78 & 4,60 & 4,55 & 2,87 & 4,78 & 3,46 & 4,00 \\
\hline \multicolumn{10}{|l|}{ Extrato 3 / 4} \\
\hline \multicolumn{10}{|l|}{ Unidade 100 PSF } \\
\hline Usuários & 2,44 & 4,48 & 4,01 & 4,33 & 4,12 & 2,29 & 2,99 & 4,22 & 3,61 \\
\hline Gestor & 1,56 & 5,00 & 3,86 & 4,46 & 4,16 & 5,00 & 4,43 & 5,00 & 4,18 \\
\hline Profissionais & 2,10 & 4,50 & 3,76 & 4,51 & 4,42 & 4,87 & 4,43 & 5,00 & 4,21 \\
\hline \multicolumn{10}{|l|}{ Unidade 268 UBS } \\
\hline Usuários & 2,89 & 3,63 & 3,99 & 4,15 & 3,90 & 1,69 & 1,62 & 4,38 & 3,28 \\
\hline Gestor & 2,67 & 5,00 & 4,29 & 5,00 & 4,79 & 3,50 & 4,86 & 4,00 & 4,26 \\
\hline Profissionais & 2,56 & 5,00 & 4,56 & 4,66 & 4,89 & 3,62 & 4,93 & 4,50 & 4,34 \\
\hline \multicolumn{10}{|l|}{ Estrato 5 / 8} \\
\hline \multicolumn{10}{|l|}{ Unidade $362 \mathrm{PSF}$} \\
\hline Usuários & 2,60 & 4,39 & 4,26 & 4,31 & 4,37 & 3,16 & 3,33 & 4,70 & 3,89 \\
\hline Gestor & 2,22 & 5,00 & 4,71 & 4,58 & 4,42 & 5,00 & 4,14 & 5,00 & 4,38 \\
\hline Profissionais & 2,88 & 4,50 & 3,64 & 3,40 & 3,45 & 4,12 & 3,50 & 4,20 & 3,71 \\
\hline \multicolumn{10}{|c|}{ Unidade 300 UBS } \\
\hline Usuários & 2,53 & 3,34 & 4,29 & 4,26 & 3,58 & 1,69 & 1,44 & 4,35 & 3,19 \\
\hline Gestor & 2,44 & 5,00 & 4,57 & 4,58 & 4,68 & 3,75 & 4,86 & 4,50 & 4,30 \\
\hline Profissionais & 2,16 & 5,00 & 3,57 & 4,21 & 4,29 & 3,25 & 2,75 & 5,00 & 3,78 \\
\hline
\end{tabular}

Negrito: $\mathrm{p}<0,05$.

O PSF também foi considerado a melhor alternativa de porta de entrada nos estratos de menor exclusão, enquanto nos de maior exclusão o indicado como o mais favorável foi o modelo tradicional representado pela UBS. Esta situação possivelmente vincula-se, por um lado, à maior densidade de equipamentos de saúde existente nas regiões de menor exclusão, o que permite aos usuários a composição de serviços no atendimento de suas necessidades; por outro, à capacidade do PSF em resolver as demandas dos usuários tal como ocorre com o modelo tradicional das UBS, que geralmente conta com médicos clínicos, pediatras e ginecologistas-obstetras. Vale salientar que, no geral, a dimensão porta de entrada apresentou altos es- cores, independentemente da modalidade analisada. Num sistema de saúde que ainda não se efetiva como universal, esta dimensão pode estar sendo superavaliada pois, por um lado, os usuários entrevistados são os que já conseguiram acessar o serviço e, por outro, os profissionais e gestores tendem a analisar a unidade a partir de referenciais exclusivamente internos, o que favorece a percepção da maior facilidade do acesso aos outros níveis de atenção. No entanto, não é isso o que ocorre de fato no cotidiano dos usuários.

A acessibilidade é percebida por usuários, profissionais e gestores como a pior dimensão nos três estratos de exclusão e nas duas modalidades de assistência básica avaliadas. Este re- 
sultado, semelhante ao encontrado por Macinko14 em Petrópolis, sugere dificuldades estruturais na oferta de serviços, para além das de organização interna às unidades. Os rígidos e limitados horários de funcionamento e as formas quase sempre presenciais de marcação de consultas funcionam como constrangimento ao efetivo acesso à atenção básica, especialmente em metrópoles, nas quais parcelas importantes da população passam o dia longe de seu local de moradia.

Para ambas as modalidades, as dimensões de porta de entrada, vínculo, serviços, coordenação e profissionais de saúde foram as mais bem avaliadas, apresentando situação próxima a 4 pontos em escala de 5 possíveis, o que configura uma situação favorável para a estruturação da atenção básica no município.

Já as dimensões acessibilidade, enfoque familiar e orientação comunitária são percebidas pelos usuários como sendo as piores, ainda que nas duas últimas o PSF destaque-se com índices ligeiramente superiores. Tal resultado sugere o questionamento do ideário oficial sobre a excelência do PSF em termos do enfoque familiar na atenção e nas atividades de promoção e educação em saúde, justamente dois elementos nucleares ao discurso da inversão do modelo assistencial em curso nas UBS.

No geral, a comparação entre as opiniões dos usuários, gestores e profissionais revela uma situação de concordância em relação ao conjunto das dimensões abordadas. Entretanto, os gestores e os profissionais tendem a anotar índices mais elevados; entre estes, os gestores são os que detêm as melhores opiniões sobre as dimensões da atenção básica. Este desempenho dos quadros profissionais, especificamente em relação ao enfoque familiar e orientação comunitária, sugere a capacidade de o PSF construir um sentimento de pertencimento a um ideário de cuidado à saúde, configurando-se em espécie de ethos para estes profissionais.

Este resultado é extremamente relevante para o planejamento da intervenção na rede, pois revela significativo grau de convergência na identificação das situações mais e menos favoráveis referentes ao conjunto das dimensões da atenção básica no município.

Em suma, a comparação entre os modelos PSF e UBS nos diferentes estratos de exclusão social mostrou que para os usuários (IAB), no geral, o PSF é superior à UBS, enquanto para os profissionais e gestores (IAB) não se revelaram diferenças relevantes entre estas duas modalidades nos três estratos de exclusão social.

Por fim, o estudo possibilitou confirmar as potencialidades do PCAT como instrumento de aferição da atenção básica à saúde da perspectiva de pelos menos três atores muito relevantes: os gestores, os profissionais e, sobretudo, os usuários - estes a própria razão de ser do sistema de saúde.

\section{Colaboradores}

P Elias e A Bousquat participaram da concepção da pesquisa, análise e discussão dos resultados, redação do artigo e aprovação da versão a ser publicada. MCG Alves participou da elaboração da metodologia, análise e discussão dos resultados e redação do artigo; CW Ferreira, da pesquisa, análise e discussão dos resultados e redação do artigo; A Cohn e A Escrivão Junior, da análise e discussão dos resultados e redação do artigo; e A Gomes e Vanessa Kishima, da pesquisa e redação do artigo 


\section{Referências}

1. Brasil. Ministério da Saúde. Datasus [acessado em 20/07/2004]. Disponível em http://www.datasus.gov.br.

2. Bousquat A, Cohn A, Elias P. O PSF e a dinâmica urbana das grandes cidades. In: Viana A L D’a, Elias P, Ibañez N, organizadores. Proteção Social: dilemas e desafios. São Paulo: Hucitec; 2005. p. 245-65.

3. Santos M. Por uma economia política da cidade -o caso de São Paulo. São Paulo: Hucitec, EDUC; 1994.

4. Lencioni S. Mudanças na metrópole de São Paulo (Brasil) e transformações industriais. Rev Departamento de Geografia 1998; 12:27-42.

5. São Paulo. Secretaria Municipal de Saúde 2005. Boletim Ceinfo-Isa Capital [acessado em 06/03/2006]. Disponível em http://www.prefeitura.sp.gov.br.

6. Brasil. Ministério da Saúde. Secretaria da Atenção à Saúde. Cadastro Nacional de Estabelecimentos [acessado em 6/03/05]. Disponível em http://cnes.datasus.gov.br.

7. Elias P, Bousquat A, Nakamura E, Cohn A. A implantação do Programa de Saúde da Família na metrópole paulista 2001-2003. Rev Bra Saúde Família 2003; 5(7):62-71.

8. Starfield B. Atenção Primária: equilíbrio entre necessidades de saúde, serviços e tecnologia. Brasília: Unesco Brasil, Ministério da Saúde; 2004.

9. Macinko J, Almeida C, Oliveira E, Sá P. Organization and delivery of primary health care services in Petrópolis, Brazil. Int J Health Plann Mgmt 2004; 19:30317.

10. Berman P. Organization of ambulatory health care provision in developing countries. Bull World Health Organ 2000; 78(6):791-802.
11. Casanova C, Colmer C. Pediatric hospitalization due to ambulatory care-sensitive conditions en Valencia (Spain). Int J Qual Health Care 1996; 8(1):51-9.

12. Starfield B. Primary care: is it essential? Lancet 1994; 344:1129-33.

13. Macinko J, Starfield B, Shi L. The contribution of primary care systems to health outcomes within organization for economic cooperation and development (OECD) countries, 1970-1998. Health Serv Res 2003; 38(4):819-54

14. Macinko J, Almeida C, Oliveira E, Sá P. Organization and delivery of primary health care services in Petrópolis, Brazil. Int J Health Plann Mgmt 2004;19:30317.

15. Macinko J, Almeida C, Oliveira E. Avaliação das características organizacionais dos serviços de atenção básica em Petrópolis: teste de uma metodologia. Rev Saúde em Debate 2003; 27(65):243-56.

16. Bousquat A, Gomes A, Cohn A, Elias P. Análise espacial da implantação do PSF no município de São Paulo. Rev C S Col 2005; 10(supl. 1). (CD dos Anais do III Congresso Brasileiro de Ciências Sociais e Humanas em Saúde).

17. Sposatti A, coordenador. Mapa da exclusão social. Revista FAPESP 2002; 83:14-20.

18. Kish, L. Survey sampling. New York: John Wiley \& Sons, Inc; 1965

19. Macinko J, Almeida C, Oliveira E, Sá P. Organization and delivery of primary health care services in Petrópolis, Brazil. Int J Health Plann Mgmt 2004;19:30317.

Artigo apresentado em 27/02/2006

Aprovado em 31/03/2006

Versão final apresentada em 17/04/2006 